\title{
Generalisations of Gödel's universe of constructible sets
}

\author{
Sy-David Friedman*
}

February 16, 2007

Gödel's universe $L$ of constructible sets has many attractive features. It has a definable wellordering (a strong form of $\mathrm{AC}$ ) and satisfies not only the generalised continuum hypothesis $(\mathrm{GCH})$, but also strong combinatorial principles such as Jensen's $\diamond, \square$ and Morass (see [6]). In this sense, the theory $\mathrm{ZFC}+V=L$ is mathematically strong.

However many interesting set-theoretic statements imply the consistency of ZFC, whereas $V=L$ does not. In this sense, the theory $\mathrm{ZFC}+V=L$ is consistency weak.

For this reason it is common in set theory to assume at least the existence of inner models of $V$ which contain large cardinals (inaccessible, measurable, strong, Woodin, superstrong and beyond). ZFC + large cardinals is consistency strong, in the sense that for an abundance of set-theoretic statements $\varphi$ (not known to be inconsistent), we have

$$
\mathrm{Con}(\mathrm{ZFC}+\mathrm{LC}) \rightarrow \mathrm{Con}(\mathrm{ZFC}+\varphi)
$$

for some large cardinal axiom LC. And in many cases, we have

$$
\mathrm{Con}\left(\mathrm{ZFC}+\mathrm{LC}^{+}\right) \rightarrow \mathrm{Con}(\mathrm{ZFC}+\varphi) \rightarrow \mathrm{Con}(\mathrm{ZFC}+\mathrm{LC})
$$

where $\mathrm{LC}^{+}$is a large cardinal axiom only slightly stronger than the large cardinal axiom LC.

${ }^{*}$ The author wishes to thank the Austrian Science Fund (FWF) for its generous support through grants P16334-NO5 and P16790-NO4 
In this article we pose the following questions.

Q1. Can we combine the mathematical power of $V=L$ with the consistency power of large cardinals?

Q2. Are large cardinals relevant solely for the calibration of consistency strengths, or do they follow from basic logical principles?

Below are some positive answers.

\section{Q1: Large cardinals and L-like models}

There are two approaches to obtaining $L$-like models with large cardinals. The first is via the

Inner model program. Show that any universe with large cardinals has an $L$-like inner model with large cardinals.

Among the important contributors to this program are Gödel, Silver, Dodd, Jensen, Mitchell, Steel and Neeman (see [1, 8, 9, 10]). We provide a hint of this work by way of several examples.

Example 1: Inaccessible cardinals

Obtaining an $L$-like inner model with an inaccessible is easy: If $\kappa$ is inaccessible, then $L \vDash \kappa$ inaccessible, and $L$ is obviously $L$-like!

Example 2: Measurable cardinals

Recall that the concept of measurable cardinal can be defined in terms of elementary embeddings. We write $j: V \rightarrow M$ to mean that $j$ is an elementary embedding from the universe $(V, \in)$ into an inner model $(M, \in)$ and $j$ is not the identity. Associated to $j$ is its critical point $\kappa$, the least ordinal $\kappa$ such that $j(\kappa) \neq \kappa$ (in fact, $j(\kappa)$ must be greater than $\kappa$ ). A cardinal is measurable iff it is the critical point of some $j: V \rightarrow M$.

We can no longer use $L$ as our desired $L$-like inner model, as in $L$ there are no measurable cardinals (a theorem of Scott). So what form should our inner model take? It will be an inner model which results from $L$ through the addition of additional predicates, in the following way. 
$A$ relativised L-hierarchy $\mathcal{L}_{\alpha}^{E}=\left(L_{\alpha}^{E}, \in, E_{\alpha}\right), \alpha \in$ Ord:

$\mathcal{L}_{0}^{E}=(\emptyset, \emptyset, \emptyset)$

$\mathcal{L}_{\alpha+1}^{E}=\left(\operatorname{Def}\left(\mathcal{L}_{\alpha}^{E}\right), \in, E_{\alpha+1}\right)\left(\right.$ in fact $\left.E_{\alpha+1}=\emptyset\right)$

$\mathcal{L}_{\lambda}^{E}=\left(L_{\lambda}^{E}, \in, E_{\lambda}\right)$, where $L_{\lambda}^{E}=\bigcup_{\alpha<\lambda} L_{\alpha}^{E}$.

Our desired inner model is $L\left[\left(E_{\alpha} \mid \alpha \in \operatorname{Ord}\right)\right]=L[E]$. But what is $E$ ? To obtain an $L$-like inner model with a measurable cardinal, the idea is to approximate the class embedding $j: V \rightarrow M$ with embeddings $E_{\lambda}$ between sets.

Theorem 1 Suppose that there is a measurable cardinal. Then there exists $E=\left(E_{\alpha} \mid \alpha \in O r d\right)$ such that:

1. For limit $\lambda, E_{\lambda}$ is either empty or an embedding $E_{\lambda}: L_{\alpha}^{E} \rightarrow L_{\lambda}^{E}$ for some $\alpha<\lambda$.

2. $L[E] \vDash$ There is a measurable cardinal.

3. $E$ is definable over $L[E]$.

4. Condensation: With mild restrictions, $M \prec \mathcal{L}_{\alpha}^{E}$ implies $M$ is isomorphic to some $\mathcal{L}_{\bar{\alpha}}^{E}$.

5. $L[E] \vDash \diamond, \square$ and (gap 1) Morass.

Property 3 gives a definable wellordering and property 4 implies GCH.

Theorem 1 has been generalised after great effort to stronger large cardinal properties (see $[8,9,10,11])$.

Why is the Inner Model Program so difficult? The verification of $L$-like properties often turns on the following principle.

Condensation: If $M$ is elementary in $\mathcal{L}_{\alpha}^{E}=\left(L_{\alpha}^{E}, \in, E_{\alpha}\right)$ then $M$ is isomorphic to some $\mathcal{L}_{\bar{\alpha}}^{E}=\left(L_{\bar{\alpha}}^{E}, \in, E_{\bar{\alpha}}\right)$.

Using Gödel's methods from $L$, it is not hard to show that $M$ as in the hypothesis of Condensation is isomorphic to some $\mathcal{L}_{\bar{\alpha}}^{F}=\left(L_{\bar{\alpha}}^{F}, \in, F_{\bar{\alpha}}\right)$. To prove Condensation, we must show that $\mathcal{L}_{\bar{\alpha}}^{F}$ equals $\mathcal{L}_{\bar{\alpha}}^{E}$. The only known technique for doing this is the comparison method, which we now describe.

Let $\bar{M}, \bar{N}$ denote $\mathcal{L}_{\bar{\alpha}}^{F}, \mathcal{L}_{\bar{\alpha}}^{E}$. Construct chains of embeddings 
$\bar{M}=\bar{M}_{0} \rightarrow \bar{M}_{1} \rightarrow \bar{M}_{2} \rightarrow \cdots \rightarrow \bar{M}_{\lambda}$

$\bar{N}=\bar{N}_{0} \rightarrow \bar{N}_{1} \rightarrow \bar{N}_{2} \rightarrow \cdots \rightarrow \bar{N}_{\lambda}$

until $\bar{M}_{\lambda}=\bar{N}_{\lambda}$. Then argue that in fact $\bar{M}=\bar{N}$.

Where do these embeddings come from?

$\bar{M}$ is of the form $\left(L_{\bar{\alpha}}^{F}, \in, F_{\bar{\alpha}}\right)$, where $F=\left\langle F_{\beta} \mid \beta<\bar{\alpha}\right\rangle$. Now choose $\beta \leq \bar{\alpha}$ so that $F_{\beta}$ is an embedding $F_{\beta}: L_{\bar{\beta}}^{F} \rightarrow L_{\beta}^{F}$ for some $\bar{\beta}<\beta$.

There is a canonical extension of $F_{\beta}$ to an embedding $F_{\beta}^{*}: L_{\bar{\alpha}}^{F} \rightarrow L_{\bar{\alpha}^{*}}^{F^{*}}$. Now adjoin the predicate $F_{\bar{\alpha}}$ to get

$$
F_{\beta}^{*}: \bar{M}=\left(L_{\bar{\alpha}}^{F}, \in, F_{\bar{\alpha}}\right) \rightarrow\left(L_{\bar{\alpha}^{*}}^{F^{*}}, \in, F_{\bar{\alpha}^{*}}^{*}\right)=\bar{M}^{*}
$$

$F_{\beta}^{*}: \bar{M} \rightarrow \bar{M}^{*}$ is the ultrapower embedding of $\bar{M}$ via $F_{\beta}$.

Thus the chains

$\bar{M}=\bar{M}_{0} \rightarrow \bar{M}_{1} \rightarrow \bar{M}_{2} \rightarrow \cdots \rightarrow \bar{M}_{\lambda}$

$\bar{N}=\bar{N}_{0} \rightarrow \bar{N}_{1} \rightarrow \bar{N}_{2} \rightarrow \cdots \rightarrow \bar{N}_{\lambda}$

are obtained by taking iterated ultrapowers.

We now come to the key question: Is $\bar{M}$ iterable, i.e., are the models $\bar{M}=\bar{M}_{0} \rightarrow \bar{M}_{1} \rightarrow \bar{M}_{2} \rightarrow \cdots \rightarrow \bar{M}_{\lambda}$ well-founded?

If so, comparison works and Condensation can be proved!

Iterability problem. Show that there are iterable structures $M=\left(L_{\alpha}^{E}, \in, E_{\alpha}\right)$ which contain large cardinals.

This has been solved up to a Woodin limit of Woodin cardinals (see [9]) and therefore there are $L$-like models with such cardinals. However it appears to be very difficult to go further.

Remark. We have greatly oversimplified the situation, for the sake of clarity. In fact, the degree of elementarity of these embeddings is a subtle issue, the extension $F_{\beta}^{*}$ of $F_{\beta}$ may have domain smaller than $L_{\bar{\alpha}}^{F}$ and moreover $\bar{M}_{i+1}$ 
may result by taking an ultrapower of some $\bar{M}_{j}$ where $j$ is less than $i$ ! This leads to "fine-structural" iterations on an "iteration tree". Such iterations are necessary for a theory of inner models for Woodin cardinals, where the serious problems with iterability begin.

We now turn to the second approach to obtaining $L$-like models with large cardinals, via the

Outer model program. Show that any model with large cardinals has an $L$-like outer model with large cardinals.

We should clarify what is meant here by "outer model". We regard the universe $V$ as a countable transitive model of GB (Gödel-Bernays class theory). Then an outer model of $V$ is a countable transitive model of GB with the same ordinals as $V$ which contains all the sets and classes of $V$. Using the method of forcing, $V$ has many outer models, as it is easy to find generic sets or classes over countable models.

The inner model program has reached Woodin limits of Woodin cardinals. But the outer model program, as I will now explain, has gone all the way!

Theorem 2 Suppose that there is a superstrong cardinal. Then there is an outer model $L[A]$ of $V$ (obtained by forcing) such that:

1. A is a class of ordinals.

2. $L[A] \vDash$ There is a superstrong cardinal.

3. $A$ is definable over $L[A]$.

4. Condensation: With mild restrictions, $M \prec\left(L_{\alpha}[A], \in, A \cap \alpha\right)$ implies $M$ is isomorphic to some $\left(L_{\bar{\alpha}}[A], \in, A \cap \bar{\alpha}\right)$.

5. $L[A] \vDash \diamond, \square$ and (gap 1) Morass.

As before, property 3 gives a definable wellordering and property 4 gives GCH.

Superstrong cardinals are much larger than Woodin cardinals. They are defined as follows.

Suppose $j: V \rightarrow M$. Recall that the critical point of $j$ is the least ordinal $\kappa$ such that $j(\kappa) \neq \kappa$. Let $\kappa$ denote the critical point of $j$. 
$j$ is $\alpha$-strong iff $V_{\alpha} \subseteq M$.

Superstrong means $j(\kappa)$-strong.

Hyperstrong means $j(\kappa)+1$-strong.

$n$-superstrong means $j^{n}(\kappa)$-strong.

$\omega$-superstrong means $j^{\omega}(\kappa)$-strong, where $j^{\omega}(\kappa)$ is the supremum of the $j^{n}(\kappa)$.

$j^{\omega}(\kappa)+1$-strong is inconsistent! (See [7].)

Thus $\omega$-superstrength is at the edge of inconsistency.

The above definitions refer to the embedding $j$. We say that a cardinal $\kappa$ is superstrong, hyperstrong, etc. iff it is the critical point of an embedding $j$ with the corresponding property.

A surprising fact is that Jensen's $L$-like principle $\square$ fails if $\kappa$ is hyperstrong (see [4]). However, we do have:

Theorem 3 With $\square$ omitted, Theorem 2 holds for $\omega$-superstrong.

Therefore it appears that the property of being $L$-like is consistent with superstrong cardinals and of being $L$-like without $\square$ is consistent with all large cardinals.

\section{Q2: The inner model hypothesis}

We now show that elementary considerations regarding the notion of consistency lead to the existence of inner models for large cardinals.

As ZFC is incomplete, there are set-theoretic statements (i.e., sentences in the language of ZFC) which, though not provable in ZFC, are nevertheless consistent with ZFC. But some statements are more consistent than others. To illustrate this, as before we regard the universe $V$ as a countable transitive model of Gödel-Bernays class theory GB. A proper class model is a transitive GB model with the same ordinals as $V$. If $M$ and $N$ are proper class models then $M$ is an inner model of $N$ iff $N$ has all of the sets an classes of $M$ and is an outer model of $N$ iff $N$ is an inner model of $M$. $M$ and $N$ are compatible iff they have a common outer model.

Definition. A statement is 
i. consistent with the ordinals iff it holds in some proper class model.

ii. consistent with $V$ iff it holds in some model compatible with $V$.

iii. internally consistent iff it holds in some inner model of $V$.

iv. externally consistent iff it holds in some outer model of $V$.

Here are some examples.

1. CH is internally consistent, as it holds in $L$, and externally consistent, as it can be forced.

2. The negation of $\mathrm{CH}$ is externally consistent, as it can be forced.

3. Con ZFC holds in all proper class models of ZFC and therefore its negation, though consistent with ZFC, is not consistent with the ordinals.

4. There are statements which are consistent with the ordinals but not internally consistent: For each ordinal $\alpha$ that is singular in $L$, let $(\beta(\alpha), n(\alpha))$ be the lexicographically least pair $(\beta, n)$ so that $\alpha$ is $\Sigma_{n+1}\left(L_{\beta}\right)$ singular. Then the sentences " $n(\kappa)$ is even for every limit cardinal $\kappa$ " and " $n(\kappa)$ is odd for every limit cardinal $\kappa$ " are both consistent with the ordinals, but they cannot both be internally consistent (see [2]).

5 . If $0^{\#}$ exists then there are statements which are consistent with the ordinals but not consistent with $V$. An example is "every limit cardinal is singular in $L "$.

Completeness for the notion of consistency

If we enlarge $V$ then it is possible that fewer statements are consistent with $V$, more statements are internally consistent and fewer statements are externally consistent.

Definition. $V$ is

i. complete for consistency iff any statement consistent with $V$ is consistent with all outer models of $V$.

ii. complete for internal consistency iff any statement true in an inner model of some outer model of $V$ is already true in an inner model of $V$.

iii. complete for external consistency iff any statement true in an outer model of $V$ is true in an outer model of any outer model of $V$.

Completeness for internal consistency is also known as the inner model hypothesis (IMH) (see [3]). In what follows we focus on the IMH; there are similar results for the notion of completeness for external consistency. 
Proposition 4 The IMH implies that $V$ is complete for consistency.

Proof. Suppose that $V$ is complete for internal consistency. If the statement $S$ is consistent with $V$ then it holds in a model compatible with $V$, i.e., in an inner model of some outer model of $V$. As $V$ is complete for internal consistency, $S$ holds in an inner model of $V$, and therefore is consistent with all outer models of $V$.

The IMH implies that there are no large cardinals in $V$ :

Theorem 5 The IMH implies that for some real $R$, there is no transitive set model of ZFC containing $R$. In particular, there are no inaccessible cardinals and the Singular Cardinal Hypothesis is true.

The IMH implies however that there are large cardinals in inner models:

Theorem 6 The IMH implies the existence of an inner model with arbitrarily large measurable cardinals (and indeed with measurable cardinals of arbitrarily large Mitchell order).

The IMH is consistent relative to large cardinals:

Theorem 7 If there is a Woodin cardinal with an inaccessible cardinal above it, then there is a countable transitive model $V$ of $G B$ which satisfies the IMH.

The strong inner model hypothesis

We conclude with a brief discussion of the strong inner model hypothesis (SIMH), a strengthening of the inner model hypothesis in which parameters are allowed. I should emphasize that no consistency proof for the SIMH from large cardinals is known, so this final section should for now be regarded as (hopefully tantalising) speculation.

The inner model hypothesis with arbitrary ordinal parameters or with arbitrary real parameters is inconsistent. To obtain a possibly consistent principle, we restrict to extensions of the universe which respect the definability and cardinality of the parameters mentioned, in the following sense. 
The hereditary cardinality of a set is the cardinality of its transitive closure. We denote the hereditary cardinality of $x$ by hcard $(x)$. Now we say that the parameter $p$ is absolute iff there is a parameter-free formula which has $p$ as its unique solution not only in $V$, but also in all outer models of $V$ with the same cardinals as $V$ up to hcard $(p)$.

Strong inner model hypothesis (SIMH). Suppose that $p$ is absolute, $V^{*}$ is an outer model of $V$ with the same cardinals up to hcard $(p)$ as $V$ and $\varphi$ is a sentence with parameter $p$ which holds in an inner model of $V^{*}$. Then $\varphi$ holds in an inner model of $V$.

If consistent, the SIMH is especially interesting, as in addition to the consequences of IMH, it provides a solution to the continuum problem:

Theorem 8 Assume the SIMH. Then $C H$ is false. In fact, $2^{\aleph_{0}}$ cannot be absolute and therefore cannot be $\aleph_{\alpha}$ for any ordinal $\alpha$ which is countable in Gödel's $L$.

It is known that the SIMH has the consistency strength of at least that of a strong cardinal, but as I have emphasized, its consistency from large cardinals remains an interesting open question.

\section{Gödel}

I end with a relevant quote from Gödel. Referring to maximum principles in set theory, he said the following:

I believe that the basic problems of abstract set theory, such as

Cantor's continuum problem, will be solved satisfactorily only

with the help of axioms of this kind.

I think that Gödel would have liked the inner model hypothesis. And he would have especially liked its stronger version ... if consistent!

\section{References}

[1] Dodd, A. The core model, London Math Society Lecture Notes 61, Cambridge University Press. 
[2] Friedman, S. Fine structure and class forcing, de Gruyter, 2000.

[3] Friedman, S. Internal consistency and the inner model hypothesis, Bulletin of Symbolic Logic, Vol.12, No.4, December 2006, pp. 591-600.

[4] Friedman, S. Large cardinals and L-like universes, in Set theory: recent trends and applications, Quaderni di Matematica, 2007.

[5] Friedman, S., Welch, P. and Woodin, H. On the consistency strength of the inner model hypothesis, submitted for publication.

[6] Jensen, R. The fine structure of the constructible hierarchy, Annals of Mathematical Logic 4, 1972.

[7] Kunen, K. Elementary embeddings and infinitary combinatorics, Journal of Symbolic Logic 36, pp. 407-413, 1971.

[8] Mitchell, W. An introduction to inner models and large cardinals, a chapter for the Handbook of Set Theory.

[9] Neeman, I. Inner models in the region of a Woodin limit of Woodin cardinals, Annals of Pure and Applied Logic, Vol. 116, pp. 67-115, 2002.

[10] Steel, J. An Outline of Inner Model Theory, in "Handbook of Set Theory", to appear.

[11] Zeman, M. Inner models and large cardinals, de Gruyter Series in Logic and Its Applications Vol.5, 2002. 